PROCEEDINGS OF THE

AMERICAN MATHEMATICAL SOCIETY

Volume 134, Number 12, December 2006, Pages 3707-3714

S 0002-9939(06)08424-3

Article electronically published on June 28, 2006

\title{
MORAVA $K$-THEORY RINGS FOR THE DIHEDRAL, SEMIDIHEDRAL AND GENERALIZED QUATERNION GROUPS IN CHERN CLASSES
}

\author{
MALKHAZ BAKURADZE AND VLADIMIR VERSHININ
}

(Communicated by Paul Goerss)

\begin{abstract}
Morava $K$-theory rings of classifying spaces of the dihedral, semidihedral and generalized quaternion groups are presented in terms of Chern classes.
\end{abstract}

\section{INTRODUCTION AND STATEMENTS}

The study of Morava $K$-theory of groups has attracted attention in the literature, for the generalized braid groups (also called Brieskorn groups or Artin groups); see for example [4].

The classifying spaces of $p$-groups with a cyclic maximal subgroup have been considered in 15, 10, 11, 12. In [11, 12] it was shown that $K(s)^{*}$ of these spaces is generated as a $K(s)^{*}(p t)$-module by Chern classes of complex vector bundles. However, the multiplicative structure in terms of Chern classes has been determined in 11] only modulo certain indeterminacy. As for [5, 10, there the multiplicative structure is given completely but in terms of artificial generators not equal to Chern classes. Our aim here is to determine the aforementioned multiplicative structure completely in terms of Chern classes by applying the formula for transfer of the first Chern class along double coverings [2], 3].

In this paper we will consider the dihedral, semidihedral and generalized quaternion 2-groups. The modular and quasidihedral groups will be considered in [1].

Let

$$
G=\left\langle a, b \mid a^{2^{m+1}}=1, b^{2}=a^{e}, b a b^{-1}=a^{r}\right\rangle, \quad m \geq 1,
$$

and either $e=0, r=-1$ (the dihedral group $D_{2^{m+2}}$ of order $2^{m+2}$ ), $e=2^{m}$, $r=-1$ (the generalized quaternion group $Q_{2^{m+2}}$ ) or $m \geq 2, e=0, r=2^{m}-1$ (the semidihedral group $S D_{2^{m+2}}$ ).

Consider the following Chern classes $c, x, c_{1}, c_{2}$ of dimensions $|c|=|x|=\left|c_{1}\right|=2$, $\left|c_{2}\right|=4$ :

$$
\begin{aligned}
& c=c_{1}\left(\eta_{1}\right), \eta_{1}: G /\langle a\rangle \cong \mathbf{Z} / 2 \rightarrow \mathbb{C}^{*}, b \mapsto-1, \\
& x=c_{1}\left(\eta_{2}\right), \eta_{2}: G /\left\langle a^{2}, b\right\rangle \cong \mathbf{Z} / 2 \rightarrow \mathbb{C}^{*}, a \mapsto-1,
\end{aligned}
$$

Received by the editors October 6, 2004 and, in revised form, July 7, 2005.

2000 Mathematics Subject Classification. Primary 55N20, 55R12, 55R40.

Key words and phrases. Transfer, formal group law, Chern class.

The first author was supported by INTAS 03-51-3251 and GRDF GEM1-3330-TB-03 grants.

The second author was supported by CNRS-NSF and INTAS 03-51-3251 grants.

(C)2006 American Mathematical Society 3707 
and $c_{i}=c_{i}\left(\xi_{\pi_{1}}\right)$, where

$$
\xi_{\pi_{1}} \rightarrow B\langle a, b\rangle
$$

is the plane bundle transferred from the canonical line bundle $\xi \rightarrow B\langle a\rangle$, for the double covering

corresponding to $\eta_{1}$.

$$
\pi_{1}: B\langle a\rangle \rightarrow B\langle a, b\rangle
$$

Theorem 1.1. i) $K(s)^{*}(B G)=K(s)^{*}\left[c, x, c_{2}\right] / R$ and the relations $R$ are determined by

(a)

$$
c^{2^{s}}=x^{2^{s}}=0
$$

$$
v_{s} c c_{2}^{2^{s-1}}=v_{s} \sum_{i=1}^{s-1} c^{2^{s}-2^{i}+1} c_{2}^{2^{i-1}}+ \begin{cases}0 & \text { if } G \text { is dihedral } \\ c^{2} & \text { if } G \text { is quaternion } \\ c x & \text { if } G \text { is semidihedral }\end{cases}
$$

(c)

and for $m>1$,

$$
v_{s}^{2} c_{2}^{2^{s}}= \begin{cases}c x+x^{2} & \text { if } G=D_{8} \\ c^{2}+c x+x^{2} & \text { if } G=Q_{8}\end{cases}
$$

$$
v_{s}^{2 \kappa(m)} c_{2}^{2^{m s}}=c x+x^{2}
$$

(d)

for $G$ of all three types,

$$
\begin{aligned}
& v_{s} x c_{2}^{2^{s-1}}=v_{s} \sum_{i=1}^{s-1} x^{2^{s}-2^{i}+1} c_{2}^{2^{i-1}}+ \begin{cases}c x+x^{2} & \text { if } G=D_{8}, \\
x^{2} & \text { if } G=Q_{8} ;\end{cases} \\
& \text { for } m>1, \\
& v_{s} x c_{2}^{2^{s-1}}=v_{s} x \sum_{i=1}^{s-1} c^{2^{s}-2^{i}} c_{2}^{2^{i-1}}+\sum_{i=1}^{m s} v_{s}^{1+\kappa(m)+2^{m s}-2^{i}} c_{2}^{\left(2^{m s}+1\right) 2^{s-1}-\left(2^{s}-1\right) 2^{i-1}} \\
&+ \begin{cases}0 & \text { if } G \text { is dihedral, } \\
c x & \text { if } G \text { is quaternion }\end{cases}
\end{aligned}
$$

where $\kappa(m)=\frac{2^{m s}-1}{2^{s}-1}$.

ii) $c^{2} x=c x^{2}, \quad c_{1}^{2^{m s}+1}=0, \quad c_{2}^{\left(2^{m s}+1\right) 2^{s-1}}=0$.

\section{Preliminaries}

Together with the covering $\pi_{1}$ we can consider the covering

$$
\pi_{2}: B\left\langle a^{2}, b\right\rangle \rightarrow B\langle a, b\rangle,
$$

corresponding to $\eta_{2}$. Then let

$$
\eta_{\pi_{2}} \rightarrow B G
$$

be the transferred line bundle associated with the double covering $\left\langle a^{4}, b\right\rangle \rightarrow\left\langle a^{2}, b\right\rangle$. The bundles $\xi_{\pi_{1}}, \eta_{\pi_{2}}$ coincide if $m=1$, but if $m>1$, then

$$
\eta_{\pi_{2}}=\left(\xi^{\otimes 2^{m-1}}\right)_{\pi_{1}} .
$$


The following bundle relations hold.

Lemma 2.1. $\quad$ i) $\eta_{i}^{\otimes 2}=\mathbb{C}, \eta_{1} \otimes \xi_{\pi_{1}}=\xi_{\pi_{1}}$;

ii) $\eta_{i} \otimes \eta_{\pi_{2}}=\eta_{\pi_{2}}$;

iii) $\eta_{\pi_{2}}^{\otimes 2}=\mathbb{C} \oplus \eta_{1} \oplus \eta_{2} \oplus \eta_{1} \otimes \eta_{2}$;

iv) $\operatorname{det} \xi_{\pi_{1}}$ is $\eta_{1}$ if $G$ is dihedral, the trivial bundle $\mathbb{C}$ if $G$ is quaternion and $\eta_{1} \otimes \eta_{2}$ if $G$ is semidihedral, and for $m>1$ one has $\operatorname{det} \eta_{\pi_{2}}=\eta_{1}$ in all three cases;

v) $\left(\left(\xi^{\otimes 2^{i}}\right)_{\pi_{1}}\right)^{\otimes 2}=\left(\xi^{\otimes 2^{i+1}}\right)_{\pi_{1}} \oplus \mathbb{C} \oplus \eta_{1}$, for $1 \leq i<m-1$. The bundle $\xi_{\pi_{1}} \otimes \xi_{\pi_{1}}=$ $\left(\xi^{\otimes 2}\right)_{\pi_{1}} \oplus\left(\xi \otimes \xi^{\otimes r}\right)_{\pi_{1}}$ is $\left(\xi^{\otimes 2}\right)_{\pi_{1}} \oplus \mathbb{C} \oplus \eta_{1}$ if $G$ is dihedral or quaternion and is $\left(\xi^{\otimes 2}\right)_{\pi_{1}} \oplus \eta_{1} \otimes \eta_{2} \oplus \eta_{2}$ if $G$ is semidihedral.

Proof. These relations are the consequences of the Frobenius reciprocity of the transfer in complex $K$-theory. For example,

$$
\begin{aligned}
\eta_{\pi_{2}}^{\otimes 2} & =\left(\xi^{2^{m-1}}\right)_{\pi_{1}}^{\otimes 2}=\left(\xi^{2^{m-1}} \otimes \pi_{1}^{*}\left(\left(\xi^{2^{m-1}}\right)_{\pi_{1}}\right)\right)_{\pi_{1}}=\left(\xi^{2^{m}} \oplus \mathbb{C}\right)_{\pi_{1}} \\
& =\eta_{2} \otimes(\mathbb{C})_{\pi_{1}} \oplus \mathbb{C} \oplus \eta_{1}=\eta_{2} \otimes\left(\mathbb{C} \oplus \eta_{1}\right) \oplus \mathbb{C} \oplus \eta_{1} .
\end{aligned}
$$

We recall the transfer formula from [3] (see also [2]).

Let $X \rightarrow X / \pi$ be a regular double covering defined by a free involution on $X$, let $\xi \rightarrow X$ be a complex line bundle, let $\xi_{\pi}$ be the transferred bundle and let

$$
\operatorname{Tr}_{\pi}^{*}: K(s)^{*}(X) \rightarrow K(s)^{*}(X / \pi)
$$

be the associated transfer homomorphism [8], [6]. Then

$$
c_{1}\left(\xi_{\pi}\right)=c_{1}(\psi)+v_{s} \sum_{i=1}^{s-1} c_{1}(\psi)^{2^{s}-2^{i}} c_{2}\left(\xi_{\pi}\right)^{2^{i-1}}+\operatorname{Tr}_{\pi}^{*}\left(c_{1}(\xi)\right),
$$

where $\psi \rightarrow X / \pi$ is the complex line bundle associated to the covering $X \rightarrow X / \pi$.

The following lemma is an easy consequence of the recursive formula for the FGL given in 4.3 .9 of [9]. See [2], Lemma 5.3.

Lemma 2.2. i) For the Honda formal group law at $p=2, s>1$, one has $F(y, z)=$ $y+z+v_{s}(y z)^{2^{s-1}}$ modulo $y^{2^{2(s-1)}}$ (or modulo $z^{2^{2(s-1)}}$ ).

ii) $F(y, z)=y+z+v_{s} \Phi\left(v_{s}, y, z\right)^{2^{s-1}}$, where $\Phi\left(v_{s}, y, z\right)=y z+v_{s}(y z)^{2^{s-1}}(y+z)$ modulo $(y z)^{2^{s-1}}(y+z)^{2^{s-1}}$.

For two line bundles with the Chern classes $y$ and $z$, respectively, $\Phi\left(v_{s}, y, z\right)$ can be regarded as the $K(s)^{*}$ orientation class of their sum.

Lemma 2.3. Let $m>1$ and either $r=-1$ or $r=2^{m}-1$. Then one has in $K(s)^{*}[u] /\left(u^{2^{(m+1) s}}\right)$,

$$
u^{2^{m s}}=\sum_{i=1}^{m s} v_{s}^{2^{m s}-2^{i}}(u[r](u))^{2^{(m+1) s-1}-\left(2^{s}-1\right) 2^{i-1}}+[r](u)(u+[r](u))^{2^{m s}-1} .
$$

Proof. The obvious decomposition in $\mathbb{F}_{2}[y, z]$,

$$
y^{2^{k}}=\sum_{i=1}^{k}(y+z)^{2^{k}-2^{i}}(y z)^{2^{i-1}}+y(y+z)^{2^{k}-1},
$$

implies for $y=u, z=[r](u), k=m s$ that

$$
u^{2^{m s}}=\sum_{i=1}^{m s}(u+[r](u))^{2^{m s}-2^{i}}(u[r](u))^{2^{i-1}}+u(u+[r](u))^{2^{m s}-1} .
$$


We want to equate the monomials

$$
(u+[r](u))^{2^{m s}-2^{i}}=(u+[r](u))^{2^{i}+\ldots+2^{m s-1}}
$$

to the monomials

$$
v_{s}^{2^{m s}-2^{i}}(u[r](u))^{\left(2^{m s}-2^{i}\right) 2^{s-1}}=v_{s}^{2^{i}+\ldots+2^{m s-1}}(u[r](u))^{\left(2^{i}+\ldots+2^{m s-1}\right) 2^{s-1}}
$$

by the equation $(u+[r](u))^{2}=v_{s}^{2}(u[r](u))^{2^{s}}$ modulo some irrelevant factor as follows.

The nilpotence degree for $u$ is $2^{(m+1) s}$, hence is $2^{(m+1) s-1}$ for $u[r](u)$. Then as it is $2^{s}$ for $F\left(u,\left[2^{m}-1\right]\right.$ ) (whereas $F(u,[-1](u))=0$ ), the nilpotence degree for $u+[r](u)$ is $2^{m s}$ by Lemma $\left.2.2 \mathrm{ii}\right)$.

Thereupon it suffices to show

$$
(u+[r](u))^{2}=v_{s}^{2}(u[r](u))^{2^{s}} \bmod (u+[r](u))^{4} .
$$

Lemma 2.2 ii) implies

$$
(u+[r](u))^{2}=v_{s}^{2}(u[r](u))^{2^{s}}+F(u,[r](u))^{2} \bmod (u+[r](u))^{2^{s}}
$$

and the dihedral and quaternion cases follow.

For the semidihedral group one has $F\left(u,\left[2^{m}-1\right](u)\right)=v_{s}^{\kappa(m)} u^{2^{m s}}$. Also, $u^{2^{m s+1}}=$ $(u[r] u)^{2^{m s}}$ as $u^{2^{m s}}=([r](u))^{2^{m s}}$. Therefore, one obtains modulo $(u+[r](u))^{2^{s}}$ ignoring powers of $v_{s}$,

$$
(u[r](u))^{2^{s}}=(u+[r](u))^{2}+(u[r](u))^{2^{m s}},
$$

$F(u,[r](u))^{2}=(u[r](u))^{2^{m s}}=\left((u+[r](u))^{2}+(u[r](u))^{2^{m s}}\right)^{2^{m s-s}}=0$ as $m s-s+1>$ $s$. The result follows.

\section{Proofs}

As mentioned in the introduction, it was proved in [11] that as a $K(s)^{*}(p t)$ module, $K(s)^{*}$ of the spaces we consider is generated by the Chern classes $c, x, c_{2}$ defined above. Let $\tilde{c_{1}}, \tilde{c_{2}}$ be the Chern classes of the bundle $\eta_{\pi_{2}}$.

Lemma 2.1 i) implies $c^{2^{s}}=0$ and $x^{2^{s}}=0$ as [2] $(c)=v_{s} c^{s^{s}}=0$ and similarly for $x$.

Let

$$
c_{1}^{*}=c_{1}+c+v_{s} \sum_{i=1}^{s-1} c^{2^{s}-2^{i}} c_{2}^{2^{i-1}}, \quad c_{1}^{* *}=\tilde{c}_{1}+x+v_{s} \sum_{i=1}^{s-1} x^{2^{s}-2^{i}} \tilde{c}_{2}^{2^{i-1}} .
$$

By (2), $c_{1}^{*} \in \operatorname{Im} \operatorname{Tr}_{\pi_{1}}^{*}, c_{1}^{* *} \in \operatorname{Im}_{\pi_{2}}^{*}$, hence $c_{1}^{2^{s}} \in \operatorname{Im}_{\pi_{\pi_{1}}}^{*}, \tilde{c}_{1}^{2^{s}} \in \operatorname{Im}_{\operatorname{Tr}_{\pi_{2}}^{*}}^{*}$ as $c^{2^{s}}=x^{2^{s}}=0$.

By the Frobenius reciprocity $c c_{1}^{*}=0$, hence by (2) $c^{2^{s-1}} c_{1}^{2^{s-1}}=0$ and $x^{2^{s-1}} c_{1}^{2^{s-1}}$ $=c^{2^{s-1}} x^{2^{s-1}}$ modulo $\operatorname{Tr}_{\pi_{1}}^{*}(u), u=c_{1}(\xi)$. From (2) one obtains $c_{1}^{2^{s-1}}=c^{2^{s-1}}$ modulo $\operatorname{Tr}_{\pi_{1}}^{*}(u)$. Hence Lemma 2.2 ii) implies modulo $\operatorname{Tr}_{\pi_{1}}^{*}(u)$,

$$
c_{1}\left(\operatorname{det} \xi_{\pi_{1}}\right)=c_{1}+v_{s} c_{2}^{2^{s-1}} \text {. }
$$

Then note $F(c, x)=c+x+v_{s} c^{2^{s-1}} x^{2^{s-1}}$, hence combining (3) and (4) we get modulo $\operatorname{Tr}_{\pi_{1}}^{*}(u)$ and $c^{2^{s-1}} x^{2^{s-1}}$,

$$
v_{s} c_{2}^{2^{s-1}}+v_{s} \sum_{i=1}^{s-1} c^{2^{s}-2^{i}} c_{2}^{2^{i-1}}= \begin{cases}0 & \text { if } G \text { is dihedral, } \\ c & \text { if } G \text { is quaternion, } \\ x & \text { if } G \text { is semidihedral. }\end{cases}
$$


Also, one has

$$
c_{1}\left(\operatorname{det} \eta_{\pi_{2}}\right)=\tilde{c}_{1}+v_{s} \tilde{c}_{2}^{s-1}+v_{s} \tilde{c}_{1}^{s} .
$$

To see (6) we need the relations ii) of Theorem 1.1. These are consequences of the relations (7)-(10).

Lemma $2.1 \mathrm{v}$ ) and (1) imply that modulo $c, x$ the Chern classes $\tilde{c}_{1}, \tilde{c}_{2}$ coincide with the first and second Chern classes of $\left(\xi_{\pi_{1}}\right)^{\otimes 2^{m-1}}$, respectively,

$$
\tilde{c}_{1}=v_{s}^{\frac{2^{(m-1) s}-1}{2^{s}-1}} c_{1}^{2^{(m-1) s}} ; \quad \tilde{c}_{2}=v_{s}^{\frac{2\left(2^{(m-1) s}-1\right)}{2^{s}-1}} c_{2}^{2^{(m-1) s}} .
$$

On the other hand, consecutively equating Chern classes of both sides of the equation in Lemma 2.1 iii) gives, respectively,

$$
\tilde{c}_{1}^{2^{s}}=c^{2^{s-1}} x^{2^{s-1}}
$$

for $m=1$,

$$
v_{2}^{2} c_{2}^{2^{s}}= \begin{cases}c x+x^{2} & \text { if } G=D_{8} \\ c^{2}+c x+x^{2} & \text { if } G=Q_{8}\end{cases}
$$

for $m>1$,

in all cases, and

$$
v_{2}^{2} \tilde{c}_{2}^{2^{s}}=c x+x^{2}
$$

$$
c^{2} x+c x^{2}=0,
$$

and we get ( (C) and relations ii). Here we use the splitting principle and write formally $\eta_{\pi_{2}}=\lambda_{1} \oplus \lambda_{2}, \quad \eta_{\pi_{2}}^{\otimes 2}=\lambda_{1}^{\otimes 2} \oplus \lambda_{2}^{\otimes 2} \oplus 2 \lambda_{1} \otimes \lambda_{2}$. Also, we take into account that the determinant $\lambda_{1} \otimes \lambda_{2}$ is known by Lemma 2.1 iv). Let $m>1, \lambda_{1} \otimes \lambda_{2}=\eta_{1}$. Then by the first equation for the Chern classes $v_{s} \tilde{c}_{1}^{2^{s}}=c+x+c+x+v_{s} c^{2^{s-1}} x^{2^{s-1}} \Rightarrow(8)$. By (7) and (国) $\tilde{c}_{1}^{2^{s}} \in \operatorname{Im} T r_{\pi_{1}}^{*}$. Also, as before, $\tilde{c}_{1}^{2^{s}} \in \operatorname{Im} T r_{\pi_{2}}^{*}$. Hence $c \tilde{c}_{1}^{2^{s}}=x \tilde{c}_{1}^{s}=0$ and by (8) $c^{i} x^{j}=0$ for $i+j>2^{s}$. Multiplying (3) by $c^{2^{s-1}} x^{2^{s-1}}$ we get $c_{1}^{2^{m s}+1}=0$; therefore, Lemma 2.2 implies $c_{2}^{\left(2^{m s}+1\right) 2^{s-1}}=0$. Then the second equation gives $v_{s}^{2} \tilde{c}_{2}^{s}+c^{2}=c x+(c+x)\left(c+x+v_{s} c^{s^{s-1}} x^{2^{s-1}}\right) \Rightarrow(9)$. The third equation gives $0=v_{s} \tilde{c}_{1}^{s} c^{2}=c x\left(c+x+v_{s} c^{2^{s-1}} x^{2^{s-1}}\right)=c x(c+x)$ and (10) follows. Similarly, for $m=1$, but $\tilde{c}_{i}=c_{i}$ and for $G=Q_{8}$ the determinant $\lambda_{1} \otimes \lambda_{2}$ is trivial.

To prove (b) for $m>1$ we raise (4) to the power $2^{m s-s}>2^{s}$. One obtains

$$
c_{2}^{2^{m s-1}}=0 \bmod \operatorname{Tr}_{\pi_{1}}^{*}(u) \text {. }
$$

By the Frobenius reciprocity of the transfer, (11) implies

$$
c c_{2}^{2^{m s-1}}=0 .
$$

Then as above $c^{i} x^{j}=0$ for $i+j \geq 2^{s}+1$. Multiplying (5) by $c$ we get (b).

Now let $m=1$. Then $\tilde{c}_{i}=c_{i}$ and $c c_{1}^{*}=0$ by (2). Hence multiplying (6) by $c$ we get (b).

Proof of (d). Let $m>1$. By the above definitions one has $\pi_{1}^{*}\left(\xi_{\pi_{1}}\right)=\xi \oplus \xi^{\otimes r}$ and $\pi^{*}\left(\eta_{2}\right)=\xi^{\otimes 2^{m}}$. Then Lemma 2.3 implies

$$
\begin{aligned}
& v_{s}^{-\kappa(m)} \pi_{1}^{*}(x)=u^{2^{m s}} \\
& =\pi_{1}^{*}\left(\sum_{i=1}^{m s} v_{s}^{2^{m s}-2^{i}} c_{2}^{2^{(m+1) s-1}-\left(2^{s}-1\right) 2^{i-1}}\right)+[r](u) \pi_{1}^{*}\left(c_{1}^{2^{m s}-1}\right) .
\end{aligned}
$$


We want to apply transfer to (13) after multiplying by $u$. By (2) $c_{1}^{2^{m s}-1} \in$ $\operatorname{ImTr_{\pi _{1}}^{*}}$ is in the annihilator of $c$, hence

$$
\operatorname{Tr}_{\pi_{1}}^{*}(u[r](u)) c_{1}^{2^{m s}-1}=\operatorname{Tr}_{\pi_{1}}^{*}(1) c_{2} c_{1}^{2^{m s}-1}=v_{s} c^{2^{s}-1} c_{2} c_{1}^{2^{m s}-1}=0
$$

and we get

$$
\operatorname{Tr}_{\pi_{1}}^{*}(u)\left(x+\sum_{i=1}^{m s} v_{s}^{\kappa(m)+2^{m s}-2^{i}} c_{2}^{2^{(m+1) s-1}-\left(2^{s}-1\right) 2^{i-1}}\right)=0
$$

Now multiplying (5) by $x+\sum_{i=1}^{m s} v_{s}^{\kappa(m)+2^{m s}-2^{i}} c_{2}^{2^{(m+1) s-1}-\left(2^{s}-1\right) 2^{i-1}}$ and using (12) and (14), the dihedral and quaternion cases follow. For the semidihedral group it remains to show that

$$
x\left(x+\sum_{i=1}^{m s} v_{s}^{\kappa(m)+2^{m s}-2^{i}} c_{2}^{2^{(m+1) s-1}-\left(2^{s}-1\right) 2^{i-1}}\right)=c x .
$$

Let us denote for ease of reading, $\Sigma=\sum_{i=1}^{m s} v_{s}^{\kappa(m)+2^{m s}-2^{i}} c_{2}^{2^{(m+1) s-1}-\left(2^{s}-1\right) 2^{i-1}}$.

Then $\Sigma(x+\Sigma)=0$ as (11) implies $\Sigma=0$ modulo $T r_{\pi_{1}}^{*}(u)$ and $\Sigma^{2}=v_{s}^{2 \kappa(m)} c_{2}^{2^{m s}}$ as the nilpotence degree of $c_{2}$ is $2^{(m+1) s-1}+2^{s-1}$. Thus $x \Sigma=v_{s}^{2 \kappa(m)} c_{2}^{2^{m s}}$ and (15) follows from (c) .

Now let $m=1$. Then $\tilde{c}_{i}=c_{i}, c c_{1}^{*}=0, x c_{1}^{* *}=0$ by (2); and by (1) det $\eta_{\pi_{2}}$ is $\eta_{1}$ (for $G=D_{8}$ ) or a trivial bundle (for $G=Q_{8}$ ). Hence multiplying (6) by $x$ we get (d).

There remains to show that the given relations give a ring of correct rank, which is

$$
2^{(m+1) s-1}+2^{2 s}-2^{s-1}
$$

according to the generalized character theory [7]. This follows by counting the obvious explicit bases of these rings according to Theorem 1.1: for $G=D_{8}$ or $Q_{8}$,

$$
\left\{c^{i} c_{2}^{j}, x^{i} c_{2}^{j}, c^{i} x c_{2}^{j}, c_{2}^{k} \mid 1 \leq i<2^{s}, 0 \leq j<2^{s-1}, 0 \leq k<2^{s}\right\} ;
$$

and for $m>1$ and all three cases,

$$
\left\{c^{i} c_{2}^{j}, c^{i} x c_{2}^{j}, c_{2}^{k} \mid 1 \leq i<2^{s}, 0 \leq j<2^{s-1}, 0 \leq k<\left(2^{m s}+1\right) 2^{s-1}\right\} .
$$

Of course there are alternative bases. For example, if one considers $c x$ as the decomposable in Theorem 1.1, then for $m>1$ the $K(s)^{*}$ base for $K(s)^{*}(B G)$ is: for $G=D_{2^{m+2}}$,

$$
\left\{c^{i} c_{2}^{j}, x^{i} c_{2}^{j}, c_{2}^{k} \mid 1 \leq i<2^{s}-j, 0 \leq j<2^{s}-1,0 \leq k<\left(2^{m s}+1\right) 2^{s-1}\right\} ;
$$

for $G=Q_{2^{m+2}},\left\{c c_{2}^{i}, x c_{2}^{i}, c_{2}^{j} \mid 0 \leq i<\left(2^{s}-1\right) 2^{s-1}, 0 \leq j<\left(2^{m s}+1\right) 2^{s-1}\right\}$; and for $G=S D_{2^{m+2}},\left\{c^{i} c_{2}^{j}, x c_{2}^{k}, c_{2}^{l} \mid 1 \leq i<2^{s}-j, 0 \leq j<2^{s}-1,0 \leq k<\left(2^{s}-1\right) 2^{s-1}\right.$, $\left.0 \leq l<\left(2^{m s}+1\right) 2^{s-1}\right\}$.

A natural question arises about the relationship between our calculations and those of [10] and 12, in terms of an alternative generating set.

The authors are grateful to Mamuka Jibladze for computer calculations of the following example, and to Björn Schuster for helpful discussions clarifying this relationship. 
Example $\left(K(2)^{*}\left(B D_{8}\right)\right)$. This example shows that the ring structures given in [10] have to be corrected. For $D_{8}$ they are correct modulo the minimal (one-dimensional) ideal, lying in the kernel of the restriction maps corresponding to all proper subgroups.

Let $A$ be the version of $K(2)^{*}\left(B D_{8}\right)$ of Theorem 1.1 and $B$ its [10] version. Then

$$
\begin{aligned}
& A=\mathbb{F}_{2}\left[v_{2}^{ \pm 1}\right]\left[c, x, c_{2}\right] / \\
& \quad\left(c^{4}, x^{4}, c^{3} c_{2}+c c_{2}^{2}, v_{2} x^{3} c_{2}+v_{2} x c_{2}^{2}+c x+x^{2}, v_{2}^{2} c_{2}^{4}+c x+x^{2}\right), \\
& B=\mathbb{F}_{2}\left[v_{2}^{ \pm 1}\right]\left[y_{1}, y_{2}, \hat{c}_{2}\right] / \\
& \quad\left(y_{1}^{4}, y_{2}^{4}, v_{2}^{2} \hat{c}_{2}^{4}+v_{2} y_{1} \hat{c}_{2}^{2}, v_{2} y_{1} \hat{c}_{2}^{2}+v_{2} y_{2} \hat{c}_{2}^{2}, v_{2} y_{2} \hat{c}_{2}^{2}+y_{1} y_{2}\right) .
\end{aligned}
$$

Choose the following basis in $A$ over $\mathbb{F}_{2}\left[v_{2}^{ \pm 1}\right]$,

$$
\begin{aligned}
\left\langle 1, c, x, c^{2}, c x, x^{2}, c_{2}, c^{3}, x^{3}, c c_{2}, x c_{2}, c x^{2}, c x^{2} c_{2}, c^{2} c_{2},\right. & \\
& \left.x^{2} c_{2}, c x c_{2}, c_{2}^{2}, c c_{2}^{2}, x c_{2}^{2}, c x c_{2}^{2}, c_{2}^{3}, x c_{2}^{3}\right\rangle
\end{aligned}
$$

and suppose there is a graded isomorphism $f: B \rightarrow A$. Then by dimension considerations,

$$
\begin{aligned}
& f\left(y_{1}\right)=\epsilon_{11} c+\epsilon_{12} x+\epsilon_{13} v_{2} c_{2}^{2}+\epsilon_{14} v_{2} x^{2} c_{2}+\epsilon_{15} v_{2} c x c_{2}+\epsilon_{16} v_{2} c^{2} c_{2}+\epsilon_{17} v_{2}^{2} x c_{2}^{3}, \\
& f\left(y_{2}\right)=\epsilon_{21} c+\epsilon_{22} x+\epsilon_{23} v_{2} c_{2}^{2}+\epsilon_{24} v_{2} x^{2} c_{2}+\epsilon_{25} v_{2} c x c_{2}+\epsilon_{26} v_{2} c^{2} c_{2}+\epsilon_{27} v_{2}^{2} x c_{2}^{3}, \\
& f\left(\hat{c}_{2}\right)=\alpha_{1} c_{2}+\alpha_{2} c^{2}+\alpha_{3} c x+\alpha_{4} x^{2}+\alpha_{5} v_{2} x c_{2}^{2}+\alpha_{6} v_{2} c c_{2}^{2}+\alpha_{7} v_{2} c x^{2} c_{2},
\end{aligned}
$$

where $\epsilon_{i j}, \alpha_{k} \in \mathbb{F}_{2}$. Then, $y_{1}^{4}=0$ implies $\left(\epsilon_{11} c+\epsilon_{12} x+\epsilon_{13} v_{2} c_{2}^{2}+\epsilon_{14} v_{2} x^{2} c_{2}+\right.$ $\left.\epsilon_{15} v_{2} c x c_{2}+\epsilon_{16} v_{2} c^{2} c_{2}+\epsilon_{17} v_{2}^{2} x c_{2}^{3}\right)^{4}=\epsilon_{13} c_{2}^{8}=\epsilon_{13} x^{2} c_{2}+\epsilon_{13} c x c_{2}+\epsilon_{13} v_{2} x c_{2}^{3}=0$, hence $\epsilon_{13}=0$.

Similarly, $y_{2}^{4}=0$ implies $\epsilon_{23}=0$.

Next, $f\left(\left(y_{1}-y_{2}\right) \hat{c}_{2}^{2}\right) c_{2}^{5}=0$ implies $\left(\epsilon_{12}+\epsilon_{22}\right) \alpha_{1} c x c_{2}^{2}=0$. Necessarily, $\alpha_{1} \neq 0$, since otherwise $c_{2}$ would not be in the image of $f$. Thus we have $\epsilon_{12}=\epsilon_{22}$. Moreover, these are not zero since otherwise $x$ would not be in the image of $f$. Thus we have

$$
\begin{aligned}
& f\left(y_{1}\right)=\epsilon_{11} c+x+\epsilon_{14} v_{2} x^{2} c_{2}+\epsilon_{15} v_{2} c x c_{2}+\epsilon_{16} v_{2} c^{2} c_{2}+\epsilon_{17} v_{2}^{2} x c_{2}^{3}, \\
& f\left(y_{2}\right)=\epsilon_{21} c+x+\epsilon_{24} v_{2} x^{2} c_{2}+\epsilon_{25} v_{2} c x c_{2}+\epsilon_{26} v_{2} c^{2} c_{2}+\epsilon_{27} v_{2}^{2} x c_{2}^{3}, \\
& f\left(\hat{c}_{2}\right)=c_{2}+\alpha_{2} c^{2}+\alpha_{3} c x+\alpha_{4} x^{2}+\alpha_{5} v_{2} x c_{2}^{2}+\alpha_{6} v_{2} c c_{2}^{2}+\alpha_{7} v_{2} c x^{2} c_{2} .
\end{aligned}
$$

Taking this into account, $f\left(y_{1} \hat{c}_{2}^{2}-y_{1} y_{2}\right) c_{2}^{2}=0$ implies $\epsilon_{11}+\epsilon_{21}+\epsilon_{24}+\epsilon_{27}=0$ and $f\left(y_{2} \hat{c}_{2}^{2}-y_{1} y_{2}\right) c_{2}^{2}=0$ implies $\epsilon_{11}+\epsilon_{21}+\epsilon_{14}+\epsilon_{17}=0$, whereas $f\left(y_{1} y_{2}-\hat{c}_{2}^{4}\right) c_{2}^{2}=0$ implies $\epsilon_{11}+\epsilon_{21}+\epsilon_{14}+\epsilon_{24}+\epsilon_{17}+\epsilon_{27}=1$. Hence $\epsilon_{11}+\epsilon_{21}=\epsilon_{14}+\epsilon_{17}=\epsilon_{24}+\epsilon_{27}=1$.

But these relations imply that $\left(f\left(y_{1}\right) f\left(\hat{c}_{2}\right)^{2}-f\left(y_{2}\right) f\left(\hat{c}_{2}\right)^{2}\right) x=c x c_{2}^{2}$, which should be actually zero as $\left(y_{1}-y_{2}\right) \hat{c}_{2}^{2}=0$.

\section{ACKNowledgments}

This work was done while the first author was holding a NATO fellowship and was visiting the University of Montpellier II (France). He is thankful for the hospitality of the Mathematical Department and especially to Professor J. Lafontaine, the director of the Institute of Mathematics and Modelization of Montpellier. The authors would like to thank the referee for his careful reading and suggestions. 


\section{REFERENCES}

1. M. Bakuradze : Morava $K$-theory rings for the modular groups in Chern classes, $K$-Theory, to appear.

2. M. Bakuradze, S. Priddy : Transfer and complex oriented cohomology rings, Algebraic \& Geometric Topology 3(2003), 473-509. MR1997326 (2004m:55007)

3. M. Bakuradze, S. Priddy: Transfered Chern classes in Morava K-theory, Proc. Amer. Math. Soc., 132(2004), 1855-1860. MR2051151(2005a:55017a)

4. K. Broto, V. V. Vershinin : On the generalized homology of Artin groups. (Russian), Zap. Nauchn. Sem. S.-Peterburg. Otdel. Mat. Inst. Steklov. (POMI) 266 (2000), Teor. Predst. Din. Sist. Komb. i Algoritm. Metody. 5, 7-12, 336; translation in J. Math. Sci. (NY) 113 (2003), no. 4, 545-547. MR1774644 (2001f:20077)

5. M. Brunetti : Morava $K$-theory of p-groups with cyclic maximal subgroups and other related p-groups, $K$-Theory 24, (2001), 385-395. MR1885128(2003a:55007)

6. A. Dold : The fixed point transfer of fibre-preserving maps, Math. Zeit. 148 (1976), 215 244. MR0433440 (55:6416)

7. M. Hopkins, N. Kuhn, and D. Ravenel : Generalized group characters and complex oriented cohomology theories, J. Amer. Math. Soc. 133 (2000), 553-594. MR1758754 (2001k:55015)

8. D. S. Kahn, S.B. Priddy: Applications of the transfer to stable homotopy theory, Bull Amer. Math. Soc. 78 (1972), 981-987. MR0309109 (46:8220)

9. D. Ravenel : Complex cobordism and Stable Homotopy Groups of Spheres, Academic Press, (1986). MR0860042 (87j:55003)

10. B. Schuster : On the Morava K-theory of some finite 2-groups, Math. Proc. Camb. Phil. Soc. 121 (1997), 7-13. MR.1418356(97i:55008)

11. M. Tezuka and N. Yagita : Cohomology of finite groups and Brown-Peterson cohomology, Algebraic Topology Arcata 1986, Springer, LNM 1370 (1989), 396-408. MR.1000392 (90i:55011)

12. N. Yagita : Complex K-theory of $B S L_{3}(Z), K$-Theory 6 (1992), 87-95. MR 1186775 (94a:19008)

Razmadze Institute of Mathematics, Tbilisi, 0193, Republic of Georgia

E-mail address: maxo@rmi.acnet.ge

Département des Sciences Mathématiques, Université Montpellier II, 34095 MontPELlier CEDEX 05, France

E-mail address: vershini@math.univ-montp2.fr

Current address: Sobolev Institute of Mathematics, Novosibirsk, 630090, Russia

E-mail address: versh@math.nsc.ru 